\title{
Strategies for Broadcasting Updates by Proactive Routing Protocols in Mobile Ad hoc Networks
}

\author{
Prince Samar and Zygmunt J. Haas \\ School of Electrical and Computer Engineering, \\ Cornell University, Ithaca, NY 14853, USA. \\ $\{$ samar, haas\}@ece.cornell.edu
}

\begin{abstract}
Proactive routing protocols for mobile ad hoc networks are table-driven, where each node tries to maintain routing information about every other node in the network at all times. Mobility of the nodes in the network causes creation of new links or breaking of old links, changing network routes dynamically. These changes require a proactive routing protocol to regularly broadcast updates in the network to enable other nodes to maintain correct routing information. The performance of a proactive protocol depends on the particular strategy the protocol uses to broadcast these updates. The updating strategies used by the current proactive protocols to broadcast detected link changes in the network are found to be inefficient. We propose three new strategies for broadcasting proactive updates in the network. Analysis of these updating schemes using simulations shows that the proposed schemes lead to significant savings in the amount of routing control traffic generated in the network. At the same time, the performance of these schemes is satisfactory in terms of the data throughput, route success rate, etc. For example, for a moderately sized network, simulation results show that the proposed schemes can lead to more than $45 \%$ savings in terms of routing control traffic, while maintaining decent performance of the network.
\end{abstract}

\section{INTRODUCTION}

Ad hoc networks are self-organizing, consisting of mobile nodes, which communicate with each other without the need of any infrastructure. Due to limitations on battery power and bandwidth, a source node communicates with a destination node using multihop routing; i.e., relying on other nodes on the path to forward its data packets.

A lot of attention has been shown by the research community to various issues related to ad hoc networks in the recent past. Many protocols have been proposed for routing in such an environment. These protocols can broadly be classified into two types: reactive and proactive routing protocols. Reactive or on-demand protocols attempt to find a route to the destination, only when the source has a packet to send to the destination. Examples are AODV, DSR, and TORA. On the other hand, proactive or table-driven protocols try to maintain routes to all the nodes in the network at all times by broadcasting routing updates in the network. Examples are DSDV [5], TBRPF [6], OLSR [7], WRP [8], STAR [9], and FSR [10].

Although reactive protocols are usually associated with less control traffic in a dynamic network, nodes have to wait until replies to the route queries are received. Also reactive

This work was supported in part by ONR under MURI contract number N00014-00-1-0564. protocols resort to frequent flooding of the network, which may cause network congestion. Proactive protocols, on the other hand, have a route to the destination readily available whenever it is needed, incurring minimal delay. But, at the same time, they may lead to a lot of wastage of the network resources if a majority of these available routes are never used.

In between the above two extremes, there are the hybrid protocols. The Zone Routing Protocol (ZRP) [1] is a hybrid proactive/reactive protocol. It is a routing framework composed of the proactive Intrazone Routing Protocol (IARP), reactive Interzone Routing Protocol (IERP), and the Bordercast Resolution Protocol (BRP). The routing zone of a node is composed of all nodes which are within "zone-radius" number of hops away from it. IERP reactively finds a route to a destination, which does not lie inside the node's zone. Any of the available reactive protocols can function as IERP [2]. The IERP uses the bordercasting mechanism of BRP [4] to find a route to the destination efficiently. IARP proactively maintains routes to all nodes belonging to its routing zone.

The Intrazone Routing Protocol (IARP) [3] uses proactive routing to maintain the routing information about the members of its zone. Proactive protocols can be classified into two primary categories: link-state and distance vector. In the link-state approach, each node maintains a view of the network topology by periodically flooding information about its outgoing links in the network. In the distance vector approach, each node maintains a list of distances to all other nodes in the network, along with the next hop to take to reach those nodes. In order to keep the distance estimates up-to-date, each node periodically broadcasts to each of its neighbors its current estimate of the shortest distance to every other node in the network. Link-state based protocols, in general, have faster convergence properties and less likelihood of having routing loops.

The performance of a proactive protocol depends on the particular strategy the protocol uses to broadcast the linkstate or the distance-vector updates to other nodes in the network. If these updates are broadcasted too often, it may lead to wastage of resources and inefficient performance of the network. On the other hand, if these updates are broadcasted too infrequently, the nodes may maintain incorrect picture of the network, leading to lost packets and routing loops.

We propose three new strategies for broadcasting updates 
by proactive routing protocols. The goal of these strategies is to reduce the amount of routing overhead in the network, while ensuring that the performance of the network does not deteriorate. This would, in turn, improve the efficiency and scalability of proactive and hybrid protocols and make them better suited for a dynamic ad hoc networking environment.

\section{RELATED WoRK}

In this section, we briefly discuss the schemes adopted by the various Mobile Ad hoc Networks (MANet) proactive routing protocols to broadcast routing updates in the network. In all proactive protocols, periodic beacons are transmitted by each node. The beacons enable a node to detect the presence of a new node within its transmit zone or a loss of a link to a neighbor.

Some of these proactive protocols broadcast routing updates periodically with a fixed interval. Probably the earliest example is the DARPA packet-radio network project [11]. The link-state based implementation of IARP in [1] periodically floods the link-state update of each node into the network. The period is inversely proportional to the maximum velocity of the nodes. The Fisheye State Routing (FSR) [10], which is a multi-scope routing protocol, uses periodic exchange of the topology map between neighbors, the frequency of which depends on the distance to a destination.

Another scheme used by proactive routing protocols is to broadcast an update for each detected change in the link status to a neighbor. This is sometimes associated with a default periodic update interval. Destination Sequenced Distance Vector (DSDV) Routing [5] proposes to use incremental updates for any change detected and a longer full periodic update interval. The Wireless Routing Protocol (WRP) [8] proposes that a node sends out an update on detecting a change in a link to a neighbor or after processing an update received from its neighbor. The Source Tree Adaptive Routing (STAR) [9] advocates broadcasting an update on detection of a new potential destination or when a known destination becoming unreachable. Updates are also broadcasted for loop-avoidance and routing table management. In Topology Broadcast based on Reverse Path Forwarding (TBRPF) [6], routing control messages are broadcasted whenever a change is detected in a selected parent/child. The Topology Control (TC) messages in the Optimized Link State Routing Protocol (OLSR) [7] are periodically broadcasted at a default interval. However, when a change occurs in the Multi-Point Relay selector set, the next TC message is broadcasted after a minimum fixed interval, starting from the time the last TC message was sent.

\section{Motivation}

Broadcasting a link-state update each time a change is detected has the potential of producing a lot of control traffic. One reason being that in a wireless environment, a radio link between mobile nodes may experience frequent disconnects and reconnects. Also, often node's link changes are correlated. For example, if a node starts moving all of a sudden, its links may break or new links may be created in quick succession. Thus, instead of broadcasting an update for each of the detected changes, if the node were to wait for a small amount of time before it broadcasted the next update, information about many changes can be conveyed in a single update packet. Hence, the node would save by broadcasting considerably smaller number of update packets, provided that a small delay can be tolerated by the network.

Consequently, broadcasting proactive updates involves a trade-off between the amount of control traffic and the consistency of the network topology information maintained by the nodes. Periodic updates are sometimes preferred in proactive protocols as they aggregate the information about link changes during the last interval in a single packet. However, broadcasting periodic updates at fixed intervals also has its own problems. Usually, the update interval is designed to reduce the delay in the worst case scenario - enabling the updates of the nodes with the most frequent changes in the network to be obtained on time by other nodes [1]. Thus the nodes which are experiencing much less frequent changes end up broadcasting a lot of redundant updates, which could be saved, if a velocity-sensitive scheme were to be used.

In order to study and optimize the trade-off between aggregation of link change updates in a single packet and the delay involved in dissemination of these link change updates, different strategies for broadcasting proactive updates are proposed in the next section. The basic structure of these strategies is as follows. In each of the strategies, a periodic update interval is found based on different assumptions and delay requirements. After each update interval, a node broadcasts an update if at least one link change was detected during the last interval. If no link changes are detected, no update is broadcasted, reducing the overhead. An update is broadcasted if the node has not broadcasted any updates in the last $M A X \_I D L E \_S L O T S$ intervals. Broadcasting an update at least every $M A X I D L E_{-} S L O T S$ is used as protection against transmission errors and loss of soft state.

\section{Proposed Strategies for Broadcasting UPDATES}

In this section, three different strategies for broadcasting proactive routing updates are proposed. These schemes determine the generation of proactive updates at a node. The relaying of these messages as they propagate through the network is done according to the rules of the particular routing protocol being used.

\section{A. Approach I: Bounded-Delay Update Strategy}

In order to decrease the routing overhead, we wish to find the largest update period for periodically broadcasting linkstate updates such that the expected delay between the detection of a link change and the broadcast of the link-state is still "small" enough. In Figure $1, T_{0}$ is the periodic update interval, the " $x$ "-es represent detection of a link change and $t_{0}$ is the delay after which the proactive update reflecting this change is broadcasted.

We assume that the arrival of a link change is an independent, identically distributed (iid) Poisson process with arrival 


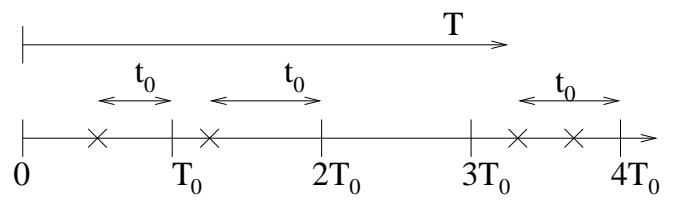

$X:$ The instance of a change in a link status

Fig. 1. Time-line showing the periodic updates after every interval $T_{0}$, the arrival of link changes and the residual time $t_{0}$ after the arrival of the link change and the time when the link-state update is broadcasted

intensity $\lambda$. This is a fair assumption to make, if the node degree is small and the nodes are moving about randomly in the network so that the process of a link being formed or broken is totally random.

Thus the problem can be formulated as follows: Maximize $T_{0}$ such that the average waiting time of all link change arrivals during the interval is bounded. In other words, maximize $T_{0}$ such that $E\left(t_{0}\right) \leq \alpha$, where $\alpha$ is the bound required on the mean value of the delay with which the update about the detected changes is broadcasted. $\alpha$ could be considered as a Quality of Service parameter of the network. Thus, the delay before which a change in the network can be reflected in the routing tables of other nodes is dependent on $\alpha$.

If in $T_{0}$ seconds no link change occurs, no update is broadcasted. Instead the node waits for another $T_{0}$ seconds. Probability that there are zero link changes in the $T_{0}$ interval is, $P(0)=1-e^{-\lambda T_{0}}$. Also, as the link change arrival is modeled as a Poisson process, given that there are $n$ link change arrivals in $T_{0}$, the $n$ unordered instants at which these changes are detected are iid uniformly distributed in $\left[0, T_{0}\right]$. Thus,

$$
\begin{aligned}
E\left(t_{0} \mid n \text { link change arrivals in } T_{0}\right) & =\int_{0}^{T_{0}} \frac{1}{T_{0}} t d t \\
& =\frac{T_{0}}{2}
\end{aligned}
$$

As $E\left(t_{0} \mid n\right.$ link change arrivals in $\left.T_{0}\right)$ is independent of $n$,

$$
E\left(t_{0}\right)=\frac{T_{0}}{2} \leq \alpha
$$

Hence the maximum value of $T_{0}$ under the constraint that the expected delay is bounded by $\alpha$ is

$$
T_{0}=2 \alpha
$$

On the basis of the above, we propose the following strategy for broadcasting updates in proactive protocols: For the performance required in the network, choose a value of $\alpha$. Set $T_{0}=2 \alpha$. After every $T_{0}$ seconds, examine if any link change has occurred within the last interval. If at least one did occur, broadcast the proactive update. Else wait for another $T_{0}$ seconds and repeat. If no changes have occurred in the last $M A X I D L E \_S L O T S$ intervals, broadcast an update.

\section{B. Approach II: Mobility-Adaptive Update Strategy}

We wish to find a periodic update interval $T_{0}$ such that the probability that one link change has occurred during this interval is maximized. This is desirable, because in this interval, it would be likely that a link change has occurred which could then be broadcasted without much delay. However, if multiple changes occur, the scheme would aggregate these changes to reduce the control traffic. In the context of the trade-off (control traffic vs network topology consistency), as discussed in section III, this scheme explores a solution which attempts to maximize the consistency of network topology using periodic updates. In practical networks, the mobility of the nodes may vary a lot over time, changing the rate at which link changes are detected. Therefore, it makes sense to have an updating strategy which is a function of the node's mobility and is, thus, adaptive.

Under the assumptions of Poisson distributed link change arrivals, the probability that one link change has occurred in $T_{0}$ is given by

$$
P\left(\text { one link change in } T_{0}\right)=\lambda T_{0} e^{-\lambda T_{0}}
$$

In order to maximize this probability, differentiate it with respect to $T_{0}$ and set it equal to 0 .

$$
\begin{aligned}
\frac{d}{d T_{0}}\left(\lambda T_{0} e^{-\lambda T_{0}}\right) & =0 \\
\Rightarrow T_{0} & =\frac{1}{\lambda}
\end{aligned}
$$

$1 / \lambda$ is the mean inter-arrival time of the link changes. This can be estimated online and the value of the update interval changed adaptively. Thus, we have a scheme for broadcasting proactive updates which is dependent on the rate of link changes of the nodes. To determine this rate of link changes, each node maintains a list to record the inter-arrival times of the last HISTORY LENGTH number of link changes for the node. The value of $T_{0}$ is set equal to the mean of these recorded inter-arrival times. This update interval changes adaptively as the network characteristics change. After every $T_{0}$ seconds, a node examines if any link change has occurred within the last interval. If at least one change did occur, the node broadcasts a proactive update. Else it waits for another $T_{0}$ seconds and repeats. Again, if no changes have occurred in the last $M A X I D L E \_S L O T S$ intervals, it broadcasts an update.

\section{Approach III: Exponential-Arrival Update Strategy}

We assume that the time of arrival of a link change is exponentially distributed with arrival intensity $\lambda$. The problem we solve here is as follows: Maximize $T_{0}$ such that the average waiting time for the first link change arrival during an update interval is bounded. If $t_{0}$ represents the delay after which the proactive update reflecting the first change during an update interval is broadcasted, the problem can be stated as: Maximize $T_{0}$ under the constraint that $E\left(t_{0}\right) \leq \alpha$, where $\alpha$ is as defined in section IV-A. Note that in Approach I, the average waiting time of all arrivals is bounded, whereas here, the average waiting time of the first arrival is bounded. In this sense, Approach III is the "worst" case, while Approach I is the "average" case. 
Let $T$ be the time at which a link change is detected by a particular node. Define $\eta=\left\lceil\frac{T}{T_{0}}\right\rceil$ and $\theta=\eta T_{0}-T$. Hence $\theta \in\left[0, T_{0}\right]$. The cumulative distribution function for the random variable $\theta$ is given by

$$
\begin{aligned}
P\left(\theta \leq t_{0}\right)= & P\left(T \epsilon\left[T_{0}-t_{0}, T_{0}\right] \cup\left[2 T_{0}-t_{0}, 2 T_{0}\right] \cup\right. \\
& \left.\cup\left[3 T_{0}-t_{0}, 3 T_{0}\right] \cup \cdots\right) \\
= & \sum_{k=1}^{\infty}\left(e^{-\lambda\left(k T_{0}-t_{0}\right)}-e^{-\lambda k T_{0}}\right) \\
= & \frac{e^{-\lambda T_{0}}}{1-e^{-\lambda T_{0}}}\left(e^{\lambda t_{0}}-1\right)
\end{aligned}
$$

Therefore, the probability density function of $\theta$ is

$$
f_{\theta}\left(t_{0}\right)=\frac{e^{-\lambda T_{0}}}{1-e^{-\lambda T_{0}}} \lambda e^{\lambda t_{0}} \quad 0 \leq t_{0} \leq T_{0}
$$

Hence the expected value of $\theta$ (or equivalently $t_{0}$ ) is given by

$$
\begin{aligned}
E\left(t_{0}\right) & =\frac{e^{-\lambda T_{0}}}{1-e^{-\lambda T_{0}}} \lambda \int_{0}^{T_{0}} t e^{\lambda t} d t \\
\Rightarrow E\left(t_{0}\right) & =\frac{T_{0}}{1-e^{-\lambda T_{0}}}-\frac{1}{\lambda} \leq \alpha
\end{aligned}
$$

This gives us a strategy for broadcasting the updates. Given the value of the bound $\alpha$ and the adaptively estimated value of the arrival rate $\lambda$, the maximum value of $T_{0}$ which satisfies (8) is calculated at the start of an update interval. Then the same algorithm as before can be used for broadcasting proactive updates in the network.

\section{Performance Evaluation}

\section{A. Simulation Set-up}

The hybrid framework of the Zone Routing Protocol was used for performance evaluation of the updating strategies. Link-state based IARP, described in [3] was used as the proactive component and a source-route based IERP described in [2] was used as the reactive component. The nodes in the routing zone of the source node form the portion of the network over which it is running the proactive protocol and its size can be changed by varying the zone radius. By using ZRP for our evaluation, we can easily emulate the different sizes of the network that use the proactive scheme. Hence, it gives us an intuition as to how the updating strategies of the proactive protocol scale, as the network size increases.

The use of a hybrid protocol may also be more practical, because proactive protocols are, in general, not very suitable for large and dynamic ad hoc networks. Their applicability in ad hoc networks is sometimes seen as a component of a hybrid routing approach like ZRP.

The network consists of 50 nodes spread randomly in an area of $1000 \times 1000$ meter $^{2}$. The transmission radius of the nodes is 225 meters. A node moves at a constant speed $v$ and is assigned an initial direction $\theta$, which is uniformly distributed between 0 and $2 \pi$. When a node reaches an edge of the simulation region, it is reflected back into the network.
As different simulation runs were performed by varying the zone radius, the network behavior was made to remain exactly the same; that is, the nodes move in exactly the same path and start sessions with exactly the same nodes at the very same instants. The simulations have been performed in the $O P N E T^{(T M)}$ simulation environment. No data was collected for the first 5 seconds to avoid measurements before the initial intrazone route discovery process stabilized. The simulation results presented here are for two different scenarios:

1. Scenario I: The 50 nodes in the network are divided into two sets of 25 nodes each. Nodes in Set I move at a speed of $15 \mathrm{~m} / \mathrm{s}$ and those in Set II move at a speed of $1 \mathrm{~m} / \mathrm{s}$. The nodes in Set I have a mean session inter-arrival delay of 10 seconds and that for Set II is 2.5 seconds. In each session, a node sends an average of 25 packets to a randomly chosen destination. The simulation length was 180 seconds. Halfway into the simulation (after 90 seconds), the two sets of nodes interchange the values of their velocity and mean session inter-arrival delay with each other - the faster moving nodes later move slowly and vice versa. This was done in order to evaluate the effects of changes in mobility and the adaptation of the proposed strategies to such changes.

2. Scenario II: Nodes in the network move at a constant speed, which is initially chosen from a uniform distribution between $0 \& 40 \mathrm{~m} / \mathrm{s}$. The mean session inter-arrival delay for each node is 5 sec. In each session a node sends an average of 25 packets to a randomly chosen destination. The simulation duration for this scenario was 90 seconds.

The optimal ZRP zone-radius for both of the scenarios was found to be 2 (see [1] for details).

The proposed schemes have been compared to the schemes used for broadcasting updates in current proactive protocols, as described in section II:

- Change-Triggered: A node broadcasts an update whenever a link change is detected. If no broadcasts have been made in the last BROADCAST_TIMEOUT $=5$ seconds, the node broadcasts an update.

- Periodic-Updates: All nodes broadcast their link-states every $B R O A D C A S T$ PERIOD seconds. This period is computed using $T_{I A R P}=\frac{3 D_{x m i t} / 20}{v_{m}}[1]$, where $v_{m}$ is the maximum velocity of the nodes in the network and $D_{x m i t}$ is the transmission radius of a node.

The parameter values chosen for the simulations are $\alpha=0.5$ seconds, MAX_IDLE_SLOTS $=10$ \& HISTORY LENGTH $=10$.

\section{B. Performance Results}

Figure 2 (a) \& (b) show the total routing control traffic generated by ZRP as a function of zone-radius for the five updating schemes, relative to the change-triggered updating scheme. As can be readily observed from the curves, the proposed strategies for broadcasting proactive updates in the network lead to significant savings in terms of the amount of control traffic generated. Studying the variation in the amount of control traffic generated as a function of zone-radius is insightful as it gives an indication about the 


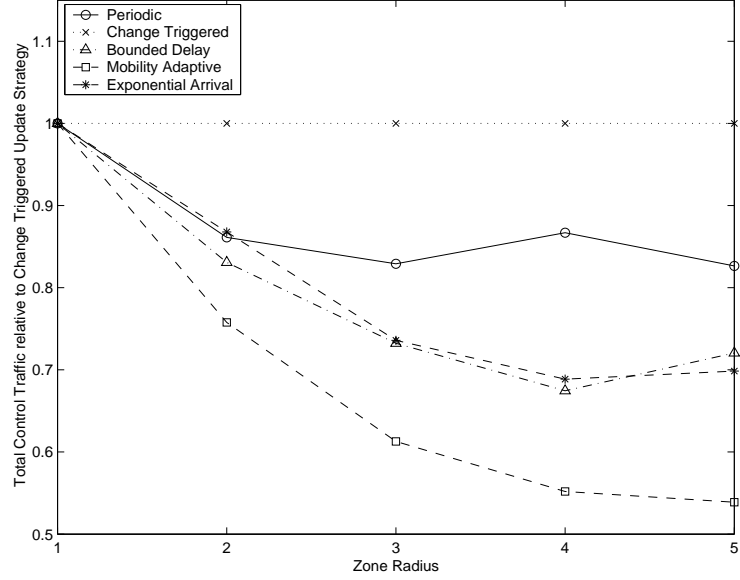

(a)

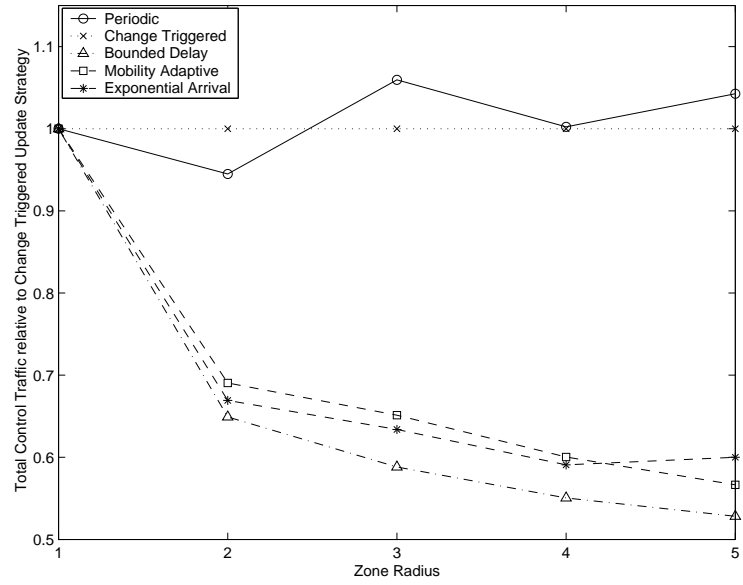

(b)

Fig. 2. The total routing control traffic (packets) generated relative to the "link change triggered" proactive update scheme, as a function of zone-radius for (a)Scenario I (b)Scenario II

scalability of the pure proactive protocol. The reduction in the routing control traffic increases as the zone-radius is increased, implying that pure proactive protocols would significantly benefit from these strategies to decrease their cost of routing. For example, in scenario I at a zone-radius of 5 , the mobility adaptive scheme reduces the amount of control traffic by about $45 \%$, as compared to the change-triggered scheme and about $35 \%$ as compared to the periodic-updating scheme. In scenario II at a zone radius of 5 , the bounded delay scheme decreases the amount of control traffic by about $47 \%$, as compared to the change-triggered scheme and about $49 \%$ as compared to the periodic scheme.

It is also observed from the curves that the mobility adaptive scheme performs better for Scenario I and the bounded delay scheme performs better for Scenario II. In Scenario I, the velocity of the nodes changes after 90 seconds. Nodes moving at $15 \mathrm{~m} / \mathrm{s}$ earlier move at $1 \mathrm{~m} / \mathrm{s}$ later and vice versa. In such a case, the mobility adaptive scheme outperforms the other schemes, as expected, because the update period of the nodes is adaptively changed as their mobility, and hence the link change rate statistics, changes. In Scenario II, where the velocity of a node remains constant throughout, the bounded delay scheme performs better. The overhead for the exponential arrival scheme is also quite small as compared to the link change triggered or the periodic updating scheme.

Figure 3 (a) \& (b) show the success rate of routing queries made by nodes in the network as a function of the zone radius for scenarios I \& II respectively. It is defined as the ratio of the total successful queries to the total number of queries made by all the nodes in the network. The reason for the apparent decrease in the route query success rate as the zone radius is increased is as follows. With a bigger zone radius, a node has proactive routes available for a larger percentage of nodes in the network, implying a reduction in the number of route queries it initiates. However, the number of unsuccessful queries due to network partitions remains the same for different zone radii, as the network conditions are made to remain the same in each case. Thus, the number of such unsuccessful queries form a bigger fraction of the total number of queries initiated by a node, leading to a decrease in the route query success rate with a increase in the zone radius. From the plots, we note that the periodic updating scheme performs the worst in terms of route query success rate. The mobility adaptive scheme performs the best in Scenario I, as the updating scheme is able to adapt well to the changing mobility of the nodes. In Scenario II, it is observed that the change triggered scheme performs the best, as any change in a node's link is immediately broadcasted in the network. In both the scenarios, the proposed schemes for broadcasting the updates perform reasonably well, with little difference between the schemes.

Figure 4 (a) \& (b) show the throughput of data packets sent by the application running at the source to that at the destination for scenarios I \& II respectively. It is defined as the ratio of the total data packets delivered to the destinations to the total data packets generated at the application of the sources in the network. Packets generated by the source's application are queued for a fixed amount of time and are discarded if no route to the destination is found in that time. The curves show that the total throughput for all the updating strategies do not vary much. The throughput for the proposed schemes is a little smaller for a zone radius of 2 or 3 , but is larger for a zone radius of 5 as compared to the change triggered scheme.

Hence, it can be inferred from the simulation results that the proposed strategies can significantly reduce the routing overhead without affecting the performance in terms of throughput and route query success rate.

\section{Conclusions}

Mobility of nodes in an ad hoc network causes creation of new links or breaking of old ones. The changes in the links of a node require a proactive routing protocol to broadcast these updates in the network. The effects of different updating schemes on the performance of a proactive or hybrid protocol have been examined. The updating strategies used by the current proactive protocols to broadcast detected link 


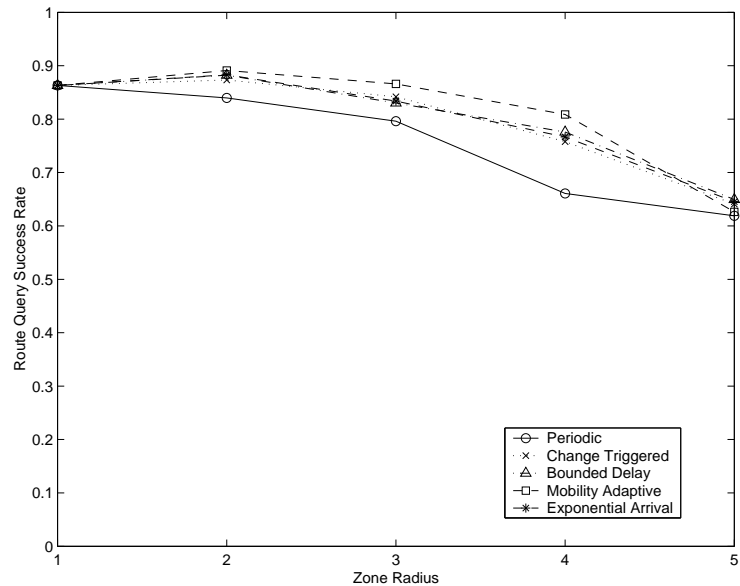

(a)

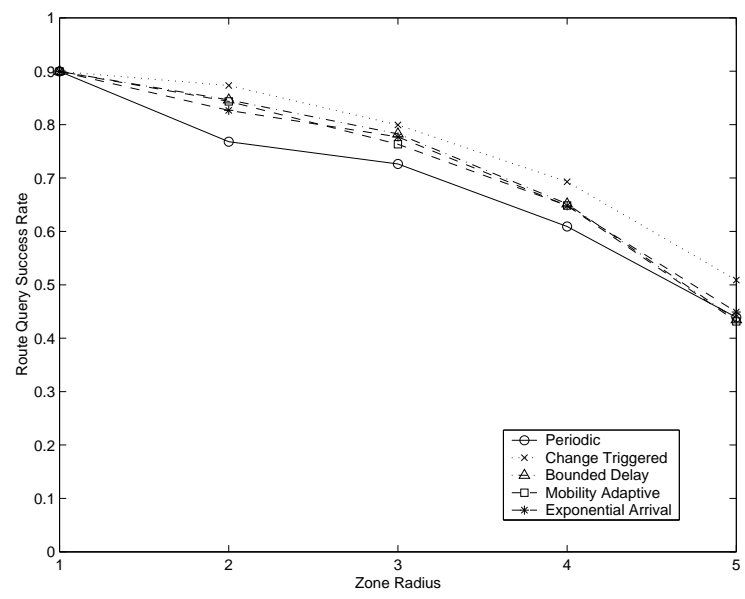

(b)

Fig. 3. The route query success rate of ZRP for the proactive update strategies as a function of zone radius for (a)Scenario I (b)Scenario II

changes are found to be inefficient in terms of the amount of routing overhead generated. We propose three new strategies for broadcasting updates in the network. Comparison of these updating schemes using simulations shows that the proposed schemes lead to significant savings in the amount of routing overhead generated in the network. At the same time, the performance of these schemes in terms of data throughput and route query success rate is close to that obtained by using the updating schemes currently in use. It is found that the mobility adaptive scheme is preferable for network where the mobility of the nodes may change. The bounded delay and exponential arrival schemes are preferable if it is required that the network be able to handle topographical changes quickly. Further, depending upon the required Quality of Service performance by the network, an appropriate value of the update period can be chosen.

\section{REFERENCES}

[1] Z.J. Haas and M.R. Pearlman, "The Performance of Query Control Schemes for the Zone Routing Protocol," ACM/IEEE Transactions on Networking, vol. 9, no. 4, pp. 427-438, August 2001.

[2] Z.J. Haas, M.R. Pearlman, and P. Samar, "The Interzone Routing

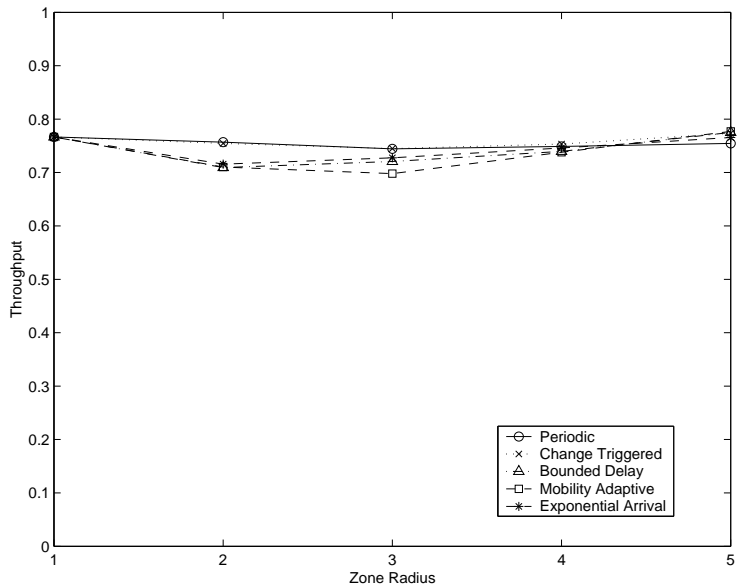

(a)

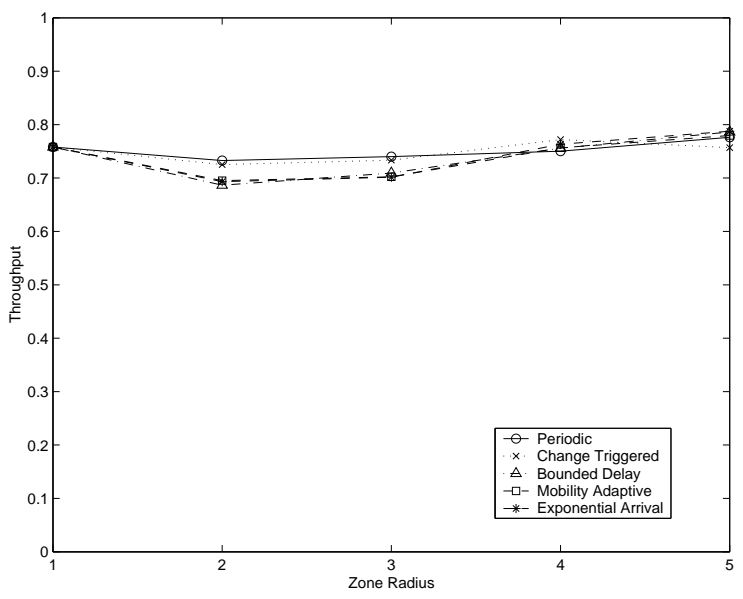

(b)

Fig. 4. The throughput of data packets as observed by the application as a function of zone-radius for (a)Scenario I (b)Scenario II

Protocol (IERP) for Ad Hoc Networks," IETF MANET, Internet Draft, July 2002.

[3] Z.J. Haas, M.R. Pearlman, and P. Samar, "The Intrazone Routing Protocol (IARP) for Ad Hoc Networks," IETF MANET, Internet Draft, July 2002.

[4] Z.J. Haas, M.R. Pearlman, and P. Samar, "The Bordercast Resolution Protocol (BRP) for Ad Hoc Networks," IETF MANET, Internet Draft, July 2002.

[5] C. Perkins and P. Bhagwat, "Highly Dynamic DestinationSequenced Distance-Vector Routing (DSDV) for Mobile Computers," in Proc. ACM SIGCOMM, October 1994

[6] B. Bellur and R.G. Ogier, "A Reliable, Efficient Topology Broadcast Protocol for Dynamic Networks," in Proc. IEEE INFOCOM, March 1999.

[7] Clausen, T., Jacquet, P., Laouiti, A., Minet, P., Muhlethaler, P., Qayyum, A., Viennot, L., "Optimized Link State Routing Protocol (OLSR)," IETF MANET, Internet Draft, October 2001.

[8] S. Murthy and Garcia-Luna-Aceves, "An Efficient Routing Protocol for Wireless Networks," Mobile Networks and Applications, 1(2), 1996, pp. 183-197.

[9] J.J. Garcia-Luna-Aceves and M. Spohn, "Efficient Routing in Packet-Radio Networks Using Link-State Information," Proc. IEEE WCNC 99, August 1999.

[10] G. Pei, M. Gerla, and T.-W. Chen, "Fisheye State Routing: A Routing Scheme for Ad Hoc Wireless Networks," Proc. ICC 2000, New Orleans, LA, June 2000.

[11] J. Jubin and J. Tornow, "The DARPA packet radio network protocols," Proceedings of the IEEE, 75(1), January 1987. 